Maria Lúcia Vaz Masson

(iD) https://orcid.org/0000-0003-0733-1753

Léslie Piccolotto Ferreira ${ }^{\mathrm{b}}$

(D) http://orcid.org/0000-0002-3230-7248

Susana Pimentel Pinto Giannini ${ }^{\mathrm{C}}$

(iD) https://orcid.org/0000-0002-1855-0655

Márcia Tiveron de Souzad

(D) https://orcid.org/0000-0002-5990-267X

Maria Maeno $^{\mathrm{e}}$

(D) https://orcid.org/0000-0001-6329-629X

Mara Edwirges Rocha Gândara ${ }^{f}$

(iD) https://orcid.org/0000-0002-7309-7021

Flávia Nogueira e Ferreira de Sousag

(D) http://orcid.org/0000-0003-0183-9361

a Universidade Federal da Bahia (UFBA), Departamento de Fonoaudiologia, Programa de Pós-Graduação em Saúde, Ambiente e Trabalho. Salvador, BA, Brasil.

b Pontifícia Universidade Católica de São Paulo (PUC-SP), Departamento de Teorias e Métodos em Fonoaudiologia e Fisioterapia, Programa de Estudos Pós-Graduados em Fonoaudiologia. São Paulo, SP, Brasil.

' Hospital do Servidor Público Municipal. São Paulo, SP, Brasil.

`Centro de Vigilância Sanitária, Divisão Técnica de Vigilância Sanitária do Trabalho, Centro de Referência em Saúde do Trabalhador. São Paulo, SP, Brasil.

e Fundação Jorge Duprat Figueiredo de Segurança e Medicina do Trabalho (Fundacentro), Centro Técnico Nacional. São Paulo, SP, Brasil.

f Universidade de São Paulo (USP), Faculdade de Medicina, Hospital das Clínicas. São Paulo, SP, Brasil.

g Ministério da Saúde, Secretaria de Vigilância em Saúde, Coordenação Geral de Saúde do Trabalhador. Brasília, DF, Brasil.

Contato:

Maria Lúcia Vaz Masson

E-mail:

masson@ufba.br

\section{Distúrbio de voz: reconhecimento revogado junto com a nova lista de doenças relacionadas ao trabalho}

\author{
Voice disorder: recognition repealed \\ together with the new work-related diseases list
}

O distúrbio de voz relacionado ao trabalho (DVRT) foi incluído na atualização da lista de doenças relacionadas ao trabalho (LDRT) do Ministério da Saúde (MS), anunciada na oportunidade da $5^{\mathrm{a}}$ Reunião da Comissão Intergestores Tripartite pela Coordenação Geral de Saúde do Trabalhador (CGSAT/MS), em 30 de julho de $2020^{1}, 21$ anos após a publicação da primeira versão, até então em vigor ${ }^{2}$. A revisão da LDRT é uma das atribuições do MS, conforme previsto na Lei $\mathrm{n}^{0} 8.080 / 1990^{3}$, no Decreto $\mathrm{n}^{0} \mathrm{7.602} / 2011^{4}$, que dispõe sobre a Política Nacional de Segurança e Saúde no Trabalho (PNSST), e na Portaria GM/MS n $n^{0} 1.823 / 2012^{5}$, a qual instituiu a Política Nacional de Saúde do Trabalhador e da Trabalhadora (PNSTT). Ao todo, foram incluídos 347 agravos na nova lista (em 1999 eram 182), a maioria deles constituída por doenças infecciosas/parasitárias (abarcando a COVID-19), seguidas por neoplasias. A nova LDRT tem objetivo clínico-epidemiológico, de identificação de agravos e doenças relacionadas ao trabalho ${ }^{1}$, e a finalidade de garantir "o desenvolvimento da atenção integral à saúde do trabalhador, com ênfase na vigilância, visando a promoção e a proteção da saúde dos trabalhadores e a redução da morbimortalidade decorrente dos modelos de desenvolvimento e dos processos produtivos" ${ }^{\prime \prime}$, conforme estabelece a PNSTT.

O DVRT caracteriza-se como uma alteração da voz decorrente do exercício profissional que diminui, compromete ou impede a atuação ou a comunicação do trabalhador, sem que haja necessariamente lesão da laringe. $\mathrm{O}$ agravo apresenta início insidioso, com piora ao final do dia, acentuando-se no decorrer dos dias. Com o repouso no final de semana, a tendência é a melhora da qualidade vocal. Contudo, o ciclo retorna com a entrada de uma nova semana, sem perspectivas de melhora ${ }^{6}$. Por ser um agravo de natureza multicausal, pode constituir-se como uma disfonia comportamental ${ }^{7}$ comum, de caráter individual, ou como um DVRT, sendo o trabalho fator contributivo ou agravante de doença preexistente ${ }^{6}$. Quando o trabalho é fator contributivo, classifica-se como Schilling II ${ }^{6,8,9}$, podendo ocorrer nas situações de disfonias funcionais ou organofuncionais (como nódulos, pólipos e edemas) provocados por fonotrauma. Na presença de uma condição preexistente, a exemplo de quadros de alergia ou mesmo de alteração estrutural mínima da cobertura da prega vocal (como nos cistos, sulcos ou pontes de mucosa), o trabalho entra como concausalidade, agravando o quadro e sendo classificado como Schilling $\mathrm{III}^{6,8,9}$.

A busca do reconhecimento do distúrbio de voz como doença relacionada ao trabalho tem o seu marco histórico em $1997^{8}$. Acolhendo pedido do Conselho Federal de Fonoaudiologia, os organizadores dos Seminários de Voz (anais disponíveis em https://www.pucsp.br/laborvox/eventos/seminario. html), da Pontifícia Universidade Católica de São Paulo (PUC-SP), deram início a um amplo debate, com a participação de diferentes atores sociais (universidades, associações e conselhos de classe, sindicatos de trabalhadores, Centros de Referência em Saúde do Trabalhador (Cerest), entidades 
assistenciais e jurídicas, dentre outros), na perspectiva de entendimento das alterações vocais como decorrentes do exercício profissional ${ }^{8-10}$. Na época, foi publicado o já revogado Decreto $\mathrm{n}^{0} 2.172 / 1997^{11}$, que regulamentava os benefícios da Previdência Social. Em seu Quadro 3 ("aparelho da fonação"), era mencionada a situação de "perturbação da palavra", mas ainda não havia métodos clínicos objetivos nem evidências que pudessem confirmar o nexo causal $^{8}$. O DVRT também não entrou na primeira LDRT ${ }^{2}$, publicada dois anos depois, em 1999. Ao longo desses 23 anos, uma agenda de iniciativas foi realizada, dentre as quais se destacam: a) a publicação da primeira versão do que veio a ser chamado Protocolo DVRT (2004), com foco na Previdência Social; b) a revisão do documento, encaminhada ao MS, com foco na notificação compulsória (2009); e c) a consulta pública realizada pelo MS (2011). Contudo, nenhuma delas foi exitosa na formalização do reconhecimento do DVRT ${ }^{8}$.

Em 2016, em um novo esforço coletivo, a Universidade Federal da Bahia (UFBA) propôs o projeto "Há evidências suficientes para reconhecer o distúrbio de voz como doença relacionada ao trabalho?”, em parceria com a PUC-SP, o Núcleo de Epidemiologia da Universidade Federal de Feira de Santana (NEPI/UEFS), o Centro Universitário Estácio de Sá, da Bahia, e a University College London. Pesquisas continuaram a ser produzidas de modo a demonstrar a relação do distúrbio de voz com o trabalho (dois manuscritos publicados neste volume da RBSO são frutos desse projeto: https://doi.org/10.1590/2317-6369000040218; https://doi.org/10.1590/2317-6369000027518), sendo retomado o contato e as discussões com a área técnica do MS. Em março de 2017, a CGSAT/MS convidou especialistas das áreas de fonoaudiologia, otorrinolaringologia, medicina do trabalho e epidemiologia, pesquisadores e representantes do órgãos de classe para participar de uma oficina para revisão do Protocolo DVRT e da ficha de notificação compulsória. O encontro contou com representantes de universidades (UFBA, UEFS, PUC-SP), Conselho Federal de Fonoaudiologia, Sociedade Brasileira de Fonoaudiologia, Associação Brasileira de Otorrinolaringologia e Cirurgia Cérvico-Facial, Academia Brasileira de Laringologia e Voz e Associação Nacional de Medicina do Trabalho, além do corpo técnico da Divisão Técnica de Vigilância Sanitária do Trabalho/Cerest Estadual de São Paulo e da CGSAT/MS, sendo a versão final do Protocolo DVRT publicada em 31 de julho de $2018^{6}$.

A continuidade do processo de revisão da LDRT culminou numa ampla consulta pública, realizada entre 28 de novembro de 2019 e 2 de fevereiro de 2020. Para um agravo/doença ser incluído na LDRT levaram-se em consideração dois critérios básicos: a) citação em duas ou mais listas internacionais consultadas pela equipe de revisores; b) o perfil epidemiológico nacional, no qual se demonstrasse a relação entre o agravo/doença com o trabalho. A versão publicada na Portaria GM/MS no 2.309, de 28 de agosto de $2020^{12}$, atualizava a LDRT, sendo composta por duas listas complementares e reversas. A ocupação/atividade econômica deixou de ser considerada, pois, além de não estar presente em todos os agravos/doenças na versão anterior, limitava o estabelecimento da relação com o trabalho. Na Lista A, constavam os agentes/fatores de risco e agravos por meio da Classificação Estatística Internacional de Doenças e Problemas Relacionados com a Saúde (CID-10). Na Lista B, ao contrário, os agravos eram demonstrados com seus respectivos agentes/fatores de risco. Para o DVRT, o principal fator de risco considerado foi o "trabalho com sobrecarga vocal", sendo aspectos agravantes os "fatores psicossociais relacionados à característica e organização do trabalho" e os "fatores relacionados ao ambiente de trabalho", os quais eram especificados nas respectivas listas. Os agravos cuja relação com o trabalho pôde ser estabelecida foram: a) laringotraqueíte aguda (J04.2); b) laringotraqueíte crônica (J37.1); c) doenças das cordas vocais e da laringe não classificadas em outra parte (J38); d) nódulos das cordas vocais (J38.2); e e) distúrbios de voz (R49). Deste modo, todo o processo de adoecimento no trabalho havia sido contemplado, partindo-se da presença dos primeiros sintomas até uma condição aguda e crônica, de modo que fosse possível identificar e intervir a qualquer momento da história natural da doença, por meio de ações integrais de atenção à saúde do trabalhador.

Um dia após sua publicação, o MS revogou a nova LDRT ${ }^{12}$, por meio da Portaria GM/MS n ${ }^{\circ} 2.345 / 2020^{13}$, tornando-a sem efeito. Na subsequente Portaria GM/MS n ${ }^{\circ} 2.384^{14}$, de 9 de setembro de 2020, dispositivos da Portaria GM/MS $n^{0} 1.339 / 1999^{15}$ são repristinados, voltando a vigorar a antiga LDRT, de 21 anos atrás. Nela, não está incluído o DVRT. Ressalta-se que a LDRT tem finalidade clínico-epidemiológica, de atenção integral à saúde, com foco na Vigilância em Saúde do Trabalhador. No caso do DVRT, a descrição facilitava a criação de uma rede de cuidado a profissionais, na sua maioria professores e teleoperadores, que historicamente adoecem em decorrência do trabalho, sofrendo impactos em sua qualidade de vida e gerando ônus para o Estado. Estimativa da Associação Brasileira de Otorrinolaringologia e Cirurgia Cérvico-Facial ${ }^{16}$, por nós atualizada, demonstra gastos na ordem de 1,6 bilhões/ano, considerando o número de professores da educação básica $(2,2 \text { milhões })^{17}$, salário em início de carreira $(\mathrm{R} \$ 2.886,24)^{18}$ e $2 \%$ de afastamentos, licenças e readaptações.

A manutenção da nova LDRT, portanto, é essencial, e também poderá atender a demais trabalhadores, que não têm a sobrecarga vocal como principal fator de risco, a exemplo da atividade de limpeza/serviços gerais e em salões de beleza (exposição a produtos químicos), do trabalho em frigoríficos (condições extremas de temperatura) e de churrasqueiros/chapeiros (exposição à fumaça) ${ }^{6}$. Por fim, a proteção à saúde de milhares de 
trabalhadores nos impõe o desafio de capacitar profissionais da Rede Nacional de Atenção Integral à Saúde do Trabalhador (Renast), que é composta por todos os serviços do Sistema Único de Saúde (SUS), em especial aqueles da atenção primária em saúde e da atenção especializada, além dos Cerest. Do mesmo modo, devem fazer parte do público a ser capacitado os profissionais de serviços privados, serviços de saúde das empresas e Serviços Especializados de Segurança e Medicina do Trabalho (SESMT), de modo a proporcionar melhores condições de trabalho, diminuição da ocorrência de distúrbios de voz e de ônus para o Estado.

\section{Agradecimentos}

Os autores gostariam de agradecer a contribuição daqueles que participaram no histórico processo de busca pelo reconhecimento do DVRT. São eles (em ordem alfabética): Ana Carolina Constantini (UNICAMP), Bernardo Bedrikow (SESI/USP/PUC-SP/UNITAU/FMSCM/HSPE-FMO/ANAMT/OIT. In memorian), Cláudia D’Oliveira (CEREST-RJ), Denise Torreão Corrêa da Silva (conselheira do CNS), Eliane Regina Zampieri de Lima (CERESTCampinas), Fernanda Figueiredo Torres (CEREST-RJ), Gustavo Polacow Korn (UNIFESP-EPM, ex-presidente ABLV), Helenice Yemi Nakamura (UNICAMP), Kionna Oliveira Bernardes Santos (UFBA), Luciano Rodrigues Neves (UNIFESP-EPM, ex-presidente ABLV), Luiz Marcello de Almeida Pereira (Estácio-FIB), Márcia Soalheiro de Almeida (CESTEH/ENSP/Fiocruz), Maria Cristina Pedro Biz (ex-conselheira CNS), René Mendes (UFMG aposentado/ USP/ ABRASTT), Silvia Ferrite (UFBA aposentada), Sílvia Maria Ramos (PUC-GO/CFFa), Tânia Maria de Araújo (UEFS). Ao corpo técnico do CGSAT/DSASTE/SVS do Ministério da Saúde, DVST/CVS - Cerest Estadual SP e Cerest Estadual RJ. Também àqueles que, por algum lapso de memória, não lembramos de citar.

\section{Referências}

1. $5^{\mathrm{a}}$ Reunião da Comissão Intergestores Tripartite [Internet]. Brasília, DF: Ministério da Saúde; 30 jun 2020 [citado em 23 ago 2020]. Video: 2:30 min. Disponível em: https://www.youtube.com/watch?v=gPCV_Fk5kKk

2. Brasil. Ministério da Saúde. Portaria ${ }^{\circ}$ 1339, de 18 de Novembro de 1999. Lista de Doenças Relacionadas ao Trabalho. Diário Oficial da União [Internet]. 19 nov 1999 [citado em 23 ago 2020]. Disponível em: https://bvsms.saude.gov.br/bvs/saudelegis/gm/1999/prt1339_18_11_1999.html

3. Brasil. Lei ${ }^{\circ} 8.080$, de 19 de setembro de 1990. Dispõe sobre as condições para a promoção, proteção e recuperação da saúde e dá outras providências. Diário Oficial da União [Internet]. 20 set 1990 [citado em 23 ago 2020]. Disponível em: https://www2.camara.leg.br/legin/fed/lei/1990/lei-8080-19-setembro-1990-365093publicacaooriginal-1-pl.html

4. Brasil. Presidência da República. Decreto $\mathrm{n}^{\circ}$ 7.602, de 7 de novembro de 2011: dispõe sobre a Política Nacional de Segurança e Saúde no Trabalho - PNSST. Diário Oficial da União [Internet]. 8 nov 2011 [citado em 23 ago 2020]. Disponível em: http://www.planalto.gov.br/ccivil_03/_ato2011-2014/2011/decreto/d7602.htm

5. Brasil. Ministério da Saúde. Portaria $n^{\circ}$ 1.823, de 23 de agosto de 2012. Institui a Política Nacional de Saúde do Trabalhador e da Trabalhadora. Diário Oficial da União [Internet]. 24 ago 2012 [citado em 23 ago 2020]. Disponível em: http://bvsms.saude.gov.br/bvs/saudelegis/gm/2012/prt1823_23_08_2012.html

6. Brasil. Ministério da Saúde. Distúrbio de voz relacionado ao trabalho - DVRT [Internet]. Brasília, DF: Ministério da Saúde; 2018 [citado em 23 ago 2020]. Disponível em: http://bvsms.saude.gov.br/bvs/publicacoes/ disturbio_voz_relacionado_trabalho_dvrt.pdf

7. Zambon F, Teixeira LC, Almeida AA. Disfonias comportamentais. In: Lopes L, Moreti F, Ribeiro LL, Pereira EC, organizadores. Fundamentos e atualidades em fonoaudiologia. Rio de Janeiro: Thieme Revinter; 2019. p. 81-93.

8. Masson MLV, Ferrite S, Pereira LMA, Ferreira LP, Araujo TM. Em busca do reconhecimento do distúrbio de voz como doença relacionada ao trabalho: movimento histórico-político. Cienc Saúde Coletiva [Internet]. 2019 [citado em 23 ago 2020];24(3):805-816. Disponível em: http://www.scielo.br/scielo.php?script=sci_ arttext\&pid=S1413-81232019000300805\&lng =en

9. Przysiezny PE, Przysiezny LTS. Distúrbio de voz relacionado ao trabalho. Braz J Otorhinolaryngol [Internet]. 2015 [citado em 23 ago 2020];81(2):202-211. Disponível em: http://www.scielo.br/scielo.php?script=sci_ arttext\&pid=S1808-86942015000200202\&lng $=$ en

10. Ferreira LP, Bernardi APA. Distúrbio de voz relacionado ao trabalho: resgate histórico. Disturb Comum [Internet]. 2011 [citado em 23 ago 2020];23(2):233-236. Disponível em: https://revistas.pucsp.br/dic/article/view/8285

11. Brasil. Decreto $\mathrm{n}^{\mathrm{O}}$ 2.172, de 5 de março de 1997. Aprova o Regulamento dos Benefícios da Previdência Social. Diário Oficial da União [Internet]. 6 mar 1997 [citado em 23 ago 2020]. Disponível em: http://www.planalto. gov.br/ccivil_03/decreto/D2172.htm 
12. Brasil. Ministério da Saúde. Portaria $\mathrm{n}^{\circ}$ 2.309, de 28 de agosto de 2020. Altera a Portaria de Consolidação n ${ }^{\circ}$ /GM/MS, de 28 de setembro de 2017, e atualiza a Lista de Doenças Relacionadas ao Trabalho (LDRT). Diário Oficial da União [Internet]. 1 set 2020 [citado em 23 ago 2020]. Disponível em: https://www.in.gov.br/en/ web/dou/-/portaria-n-2.309-de-28-de-agosto-de-2020-275240601

13. Brasil. Ministério da Saúde. Portaria MS/GM n ${ }^{\circ}$ 2.345, de 02 de setembro de 2020. Torna sem efeito a Portaria $\mathrm{n}^{\mathrm{o}}$ 2.309/GM/MS, de 28 de agosto de 2020. Diário Oficial da União [Internet]. 2 set 2020 [citado em 3 set 2020 ]. Disponível em: https://www.in.gov.br/en/web/dou/-/portaria-n-2.345-de-2-de-setembro-de-2020-275488423

14. Brasil. Ministério da Saúde. Portaria MS/GM n 2.384, de 09 de setembro de 2020. Repristina os arts. 423 e 424 da Seção IV do Capítulo III do Título III e o Anexo LXXX da Portaria de Consolidação no 5/GM/MS, de 28 de setembro de 2017. Diário Oficial da União [Internet]. 9 set 2020 [citado em 15 set 2020]. Disponível em: https:// www.in.gov.br/web/dou/-/portaria-n-2.384-de-8-de-setembro-de-2020-276379302

15. Brasil. Ministério da Saúde. Portaria MS/GM no 1.339, de 18 de novembro de 1999. Institui a Lista de Doenças relacionadas ao Trabalho. Diário Oficial da União [Internet] 18 nov 1999 [citado em 15 set 2020]. Disponível em: http://bvsms.saude.gov.br/bvs/saudelegis/gm/1999/prt1339_18_11_1999.html

16. Associação Brasileira de Otorrinolaringologia e Cirurgia Cérvico-Facial. Carta do Rio, aprovada no Consenso de Voz Profissional, é finalizada [Internet]. São Paulo: Associação Brasileira de Otorrinolaringologia e Cirurgia Cérvico-Facial; 1 set 2004 [citado em 3 ago 2020]. Disponível em: http://www.ablv.com.br/imageBank/ Consenso-2004-Relatorio-Final.pdf

17. Brasil. Instituto Nacional de Estudos e Pesquisas Educacionais Anísio Teixeira. Censo da Educação Básica 2019: resumo técnico [Internet]. Brasília, DF: Ministério da Educação; 2020 [citado em 3 ago 2020]. Disponível em: https://bit.ly/3n0tugL

18. Brasil. Ministério da Educação. MEC divulga reajuste do piso salarial de professores da educação básica para 2020 [Internet]. Brasília, DF: Ministério da Educação; 17 jan 2020 [citado em 3 ago 2020]. Disponível em: https://bit.ly/2S7iCja 